duration was $156(48-420)$ months. DAS 28 score was $2.86(0.68-5.70)$ and $54.5 \%$ of the patients were in remission. BMOD synovitis, erosion and PDUS synovitis total scores were $20(6-36) ; 6(0-17) ; 1(0-14)$ respectively. Although US findings were not correlated with DAS 28 and grip strength; there was poor correlation between US findings and DASH, RAAD and disease duration (Table 1). Signs of synovitis indicated with PDUS in $63.3 \%$ of the joints assessed. High-grade PDUS signal (grade 3) was found in $10(22.7 \%)$ of the patients. Duration of morning stiffness, $\mathrm{HDI}$ and DASH scores were worse in the patients with high-grade PDUS signals ( $p=0.01,0.04,0.01$ respectively)

Table 1. Correlation coefficients between clinical, ultrasound and functional variables

\begin{tabular}{|c|c|c|c|c|c|c|}
\hline & \multicolumn{2}{|c|}{$\begin{array}{l}\text { BMOD synovitis } \\
\text { Total }\end{array}$} & \multicolumn{2}{|c|}{$\begin{array}{l}\text { BMOD erosions } \\
\text { Total }\end{array}$} & \multicolumn{2}{|c|}{$\begin{array}{l}\text { PDUS synovitis } \\
\text { Total }\end{array}$} \\
\hline & $r$ & $p$ & $r$ & $p$ & $r$ & $\mathrm{P}$ \\
\hline DASH & 0.37 & $0.02^{\star}$ & 0.19 & 0.20 & 0.13 & 0.38 \\
\hline $\mathrm{HDI}$ & 0.21 & 0.17 & 0.17 & 0.28 & 0.21 & 0.17 \\
\hline RAAD & 0.33 & $0.02^{*}$ & 0.41 & $0.00^{*}$ & 0.22 & 0.15 \\
\hline DAS 28 & -0.04 & 0.78 & 0.01 & 0.95 & -0.05 & 0.75 \\
\hline MS & 0.24 & 0.12 & -0.10 & 0.51 & 0.20 & 0.19 \\
\hline Disease duration & 0.13 & 0.38 & 0.46 & $0.00^{*}$ & -0.31 & $0.03^{*}$ \\
\hline Lateral GS & 0.07 & 0.64 & -0.06 & 0.69 & -0.06 & 0.70 \\
\hline Tip GS & 0.11 & 0.49 & -0.27 & 0.08 & 0.09 & 0.56 \\
\hline Three fingered GS & 0.21 & 0.89 & -0.17 & 0.27 & 0.05 & 0.76 \\
\hline Mass grasp & -0.08 & 0.59 & -0.078 & 0.61 & -0.12 & 0.43 \\
\hline
\end{tabular}

BMOD: B mode ultrasound, PDUS: Power Doppler ultrasound, DASH: Disabilities of the Arm, Shoulder and Hand, HDI: Hand disability index, RAAD: Rheumatoid Arthritis Articular Damage, DAS 28: Disease activity score, MS: Morning stiffness, GS: Grip strength. ${ }^{*} \mathrm{P}<0.05$.

Conclusions: US scores in established RA patients are usually high because of synovial hypertrophy. It is considered that the high grade PDUS signals are more appropriate for evaluation of long-standing RA patients. Furthermore in this study, grade 3 PDUS signals were found to be a good indicator of synovial inflammation, morning stiffness, and disability.

References:

[1] Concordance between Clinical and ultrasound findings in rheumatoid arthritis. Garrigues F, Jousse-Joulin S, Bouttier R et al. Joint Bone Spine 80 (2013) 597-603.

[2] Severity and Diurnal Improvement of Morning Stiffness Independently Associate with Tenosynovitis in Patients with Rheumatoid Arthritis. Kobayashi Y, Ikeda K, Nakamura T et al. Plos One (11) Doi: 10.1371/journal.pone.0166616. Disclosure of Interest: None declared

DOI: 10.1136/annrheumdis-2017-eular.2898

\section{AB0214 METHOTREXATE IN EARLY RHEUMATOID ARTHRITIS: A SINGLE-CENTER EVALUATION OF CLINICAL OUTCOME COMPARING TWO STARTING TREATMENT STRATEGIES}

F. Benaglio, S. Bugatti, G. Sakellariou, G. Crepaldi, G. Zanframundo, C. Montecucco, R. Caporali. Early Arthritis Clinic, Division of Rheumatology, University of Pavia, IRCCS Policlinico San Matteo Foundation, Pavia, Italy

Background: methotrexate (MTX) is considered the 'anchor drug' in the treatment of rheumatoid arthritis (RA) and it should be part of the first treatment strategy: there are many indications about the correct way to optimize the dosage, depending on clinical response and tolerability, but currently there is no shared evidence about the optimal starting dosage.

Objectives: to compare two different starting treatment strategies with MTX in patients with early RA evaluated at our Early Arthritis Clinic (EAC) in order to assess the rate of patients who reaches the target (remission/low disease activity) at 6 months, according with EULAR guidelines.

Methods: patients with RA (disease duration $<12$ months) evaluated at our EAC between 2005 and 2016 and treated with MTX parenterally and glucocorticoids (GC) were included. Patients followed a treat-to-target strategy to reach low disease activity with bimonthly tight control. Patients evaluated between 2005 and 2009 were initially treated with MTX $10 \mathrm{mg} /$ week + GC (group A) with increase of MTX to 15 and then $20 \mathrm{mg} /$ week in case of failure to reach the target; patients evaluated between 2010 and 2016 were initially treated with MTX 15 mg/week + GC (group B) with possible increase to 20 and $25 \mathrm{mg} /$ week. The DAS28 response was assessed after 6 months.

Results: 260 patients were analyzed: 123 in group A vs 137 in group B. At baseline patients showed differences in DAS28 $(5.2 \pm 1.15$ vs $4.6 \pm 1.16, p<0.0001)$ and $\mathrm{HAQ}(1,125$ vs 1 IQR IQR $0.75-1.8750 .375-1.5, \mathrm{p}=0.006)$; there were no differences in terms of autoimmunity. After 6 months of therapy there were no differences in clinical response: $32 \%$ of patients in the group $A$ reached the DAS28 remission vs $40 \%$ of the group B ( $p=n s), 27 \%$ in the group A reached the DAS28 low disease activity vs $24 \%$ of the group B $(p=n s), 41 \%$ of the group A was in moderate disease activity vs $36 \%$ in the group $B(p=n s)$. The need to increase the dosage of MTX during the first 6 months was similar: $27.6 \%$ of the group A vs $29.9 \%$ of the group B ( $p=n s)$, conversely, the need to reduce the dosage of MTX due to intolerance and/or adverse event was significantly higher in the group B (group A: $1.6 \%$ vs group B: $9.5 \%, p=0.014$ ).

Conclusions: the use of higher dose of MTX is associated with a higher rate of side effects but does not provide, at short term, a significant improvement in term of clinical outcome. Conversely, the initial use of MTX $10 \mathrm{mg} / \mathrm{week}$, with a quick dose titration in case of persistent disease activity, seems to be an appropriate option for many patients with early RA, as also recently suggested by the Utrecht Arthritis Cohort Study Group; in spite of this, there is an amount of patients who do not achieve the clinical target at short term regardless of the initial dose of MTX. Our experience suggests that in the early phase of RA treatment, in a context based on early diagnosis, tight control and treat to target, the clinical outcome seems to be linked more to the treatment strategy than to the drug dosage used. References:

[1] Combe B, et al. 2016 update of the EULAR recommendations for the management of early arthritis. Ann Rheum Dis-2016-210602.

[2] Nair SC, et al. Determining the lowest optimally effective methotrexate dose for Individual RA patients using their dose response relation in a tight control treatment approach. PLoS ONE 11:e0148791.

Disclosure of Interest: None declared

DOI: 10.1136/annrheumdis-2017-eular.6437

\section{AB0215 A LONGER MEAN SURVIVAL OF BIOLOGIC TREATMENTS IS ASSOCIATED WITH DAS28 REMISSION IN RHEUMATOID ARTHRITIS PATIENTS}

F. Ometto, C. Botsios, B. Raffeiner, D. Astorri, L. Friso, L. Bernardi, L. Punzi, A. Doria. Rheumatology Unit, Department of Medicine - Dimed, University of Padova, PADOVA, Italy

Objectives: To determine factors associated with remission (DAS28<2.6). We specifically considered the association of biologic treatment duration, number of biologic switches and survival of biologic treatment with remission.

Methods: We conducted a retrospective analysis on a monocentric cohort of rheumatoid arthritis patients. We included patients who were on biologic drugs at the time of the analysis (31st December 2016). We considered patients starting the first biologic treatment since January 2000 and with a follow-up $\geq 12$ months. We considered the following variables: demographics, positive rheumatoid factor (RF)/ anti-citrullinated peptides (ACPA), disease duration at the start of the first biologic treatment, number of biologic switches, clinical assessment at the last follow-up, concomitant DMARDs, prednisone dose, current biologic treatment. We also considered the mean survival of biologic treatments, defined as the duration of the biologic treatment of each patient divided by the number of biologics undergone by the patient.

Mann-Whitney test and chi-square test were used to assess the association of continuous and categorical variables with the outcome. Continuous measures are reported as medians and interquartile range. Multivariate regression analysis included all variables reaching a $p$ value $<0.2$ in univariate analysis

Results: We collected data of 330 patients. All patients had complete data. One hundred thirty-five patients $(40.9 \%)$ were in DAS28 remission. Characteristics of the patients are reported in Table I. We considered 609 biologic treatments (abatacept $n=61$; anti-TNF alpha $n=445$; anakinra $n=43$; tocilizumab $n=56$; rituximab $n=5$ ). Total biologic treatment duration in all patients was 9.62 years (5.68-12.53), in patients in remission 8.95 (4.70-12.53) and in patients not in remission $10.12(6.28-12.62)(p=0.248)$. Median number of previous biologic switches was $1.00(0-1.00)$ in patients in remission and not in remission ( $p=0.436)$. Survival of biologic treatments was 5.33 years $(2.89-7.72)$ in all patients, 4.57 (2.54-7.28) in patients in remission and 5.81 (3.49-7.93) in patients not in remission $(p=0.013)$.

All clinical assessments at the last follow-up were significantly associated with DAS28 remission. Mean prednisone dose was significantly lower in patients in DAS28 remission but was considered as a consequence of remission rather than a predictor (Table I). The type of current biologic treatment was not significantly associated with DAS28 remission.

Variables included in the multivariate regression analysis were: BMI, age, disease duration, positive RF/ACPA mean survival of each biologic treatment. All variables included in the model were independently associated with DAS28 remission. A higher BMI, older age, longer disease duration and positive RF/ACPA were negatively associated with remission. A longer mean survival of biologic

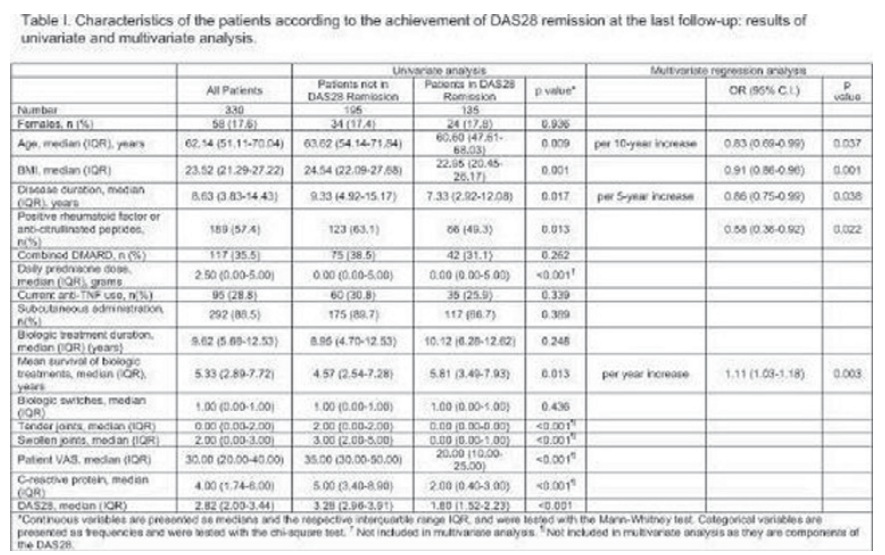


treatments was positively associated with DAS28 remission (OR 1.11 per year increase, $95 \%$ C.I. $1.03-1.18, p=0.003)$.

Conclusions: The duration of biologic treatment and the number of previous biologic switches were not associated with of DAS28 remission. Indeed, a longer survival of biologic treatments was associated with remission. The mean survival of biologic treatments reflects both a smaller number of biologic failures and a prolonged response to each biologic treatment.

Disclosure of Interest: None declared

DOI: 10.1136/annrheumdis-2017-eular.4482

\section{AB0216 EFFECTS OF ANTI-CITRULLINATED PROTEIN ANTIBODIES ON} SYSTEMIC BONE MASS IN RHEUMATOID ARTHRITIS PATIENTS

G. Orsolini, C. Caimmi, O. Viapiana, L. Idolazzi, E. Fracassi, D. Gatti, G. Adami, M. Rossini. Rheumatology Unit, University of Verona, Verona, Italy

Background: Bone loss in rheumatoid arthritis (RA) is a key feature both local and systemic. Anti-citrullinated protein antibodies (ACPA) have recently been found to directly induce differentiation and activation of osteoclasts and therefore contribute to periarticular bone loss.

Objectives: The aim of this study was to analyze the effect of ACPA on systemic bone mineral density (BMD) in patients with established RA.

Methods: This is a cross-sectional study with a single-center RA population. $B M D$ was measured with Dual X-ray absorptiometry at lumbar and femoral sites. ACPA were measured by EIA. Multivariate analysis was performed adjusting for the main confounding variables.

Results: One hundred twenty-seven RA patients were enrolled. In univariate analysis ACPA-positive patients showed lower BMD Z-score (SD below the ageand gendermatched mean reference value) at femoral sites $(p<0.01)$. A negative correlation between ACPA titer and BMD Z-score at all sites was observed $(p<0.01)$. The multivariate analysis adjusted for the main confounding variables confirmed the negative effect of ACPA at femoral sites $(p<0.05)$, but not at lumbar spine BMD. No significant effect of rheumatoid factor has been observed.

Conclusions: ACPA have a negative titer-dependent effect on BMD at femoral sites, mainly constituted by cortical bone. ACPA-positive patients, especially if at high titer, should undergo bone investigations and be treated with bone protecting agents. Disease-modifying anti-rheumatic drugs lowering ACPA titer might have positive effects on systemic bone mass.

Disclosure of Interest: None declared

DOI: 10.1136/annrheumdis-2017-eular.2672

\section{AB0217 THE PROGNOSTIC VALUE OF IGA AUTOANTIBODIES (RHEUMATOID FACTOR AND ACPA) FOR PREDICTION OF THERAPEUTIC RESPONSES TO ANTI-TNF THERAPY IN PATIENTS WITH RHEUMATOID ARTHRITIS}

G. Steiner ${ }^{1}$, D. Sieghart ${ }^{1}$, F. Alasti ${ }^{1}$, P. Studenic ${ }^{1}$, T. Perkmann ${ }^{2}$, T. Horn ${ }^{3}$, D. Aletaha ${ }^{1}$, J.S. Smolen ${ }^{1} .{ }^{1}$ Internal Medicine III-Rheumatology; ${ }^{2}$ Laboratory Medicine, Medical University Vienna; ${ }^{3}$ Phadia Austria GmbH, Thermo Fisher Scientific, Vienna, Austria

Background: Anti-citrullinated protein antibodies (ACPA) and rheumatoid factor (RF) are important diagnostic markers in rheumatoid arthritis (RA). These antibodies are predominantly of the IgM (RF) or IgG (ACPA) isotype. The added diagnostic and prognostic value of $\lg A$ autoantibodies is being debated.

Objectives: To determine the prevalence of $\lg A-R F$ and $\lg A-A C P A$ in patients with RA and to investigate their potential predictive value regarding response to treatment with methotrexate (MTX) and TNF inhibitors.

Methods: A total of 255 patients were tested for the presence of IgA-RF, IgA-ACPA and IgG-ACPA by Eli $A^{\circledR}$ (Thermo Fisher Scientific); IgM-RF was measured by nephelometry. Therapeutic responses to MTX and TNF blocking biologicals were calculated in an inception cohort $(n=104)$ who had started their DMARD therapy at our clinic. To define therapeutic responses simplified disease activity index (SDAI) 50 and American College of Rheumatology (ACR) 20 responses were calculated. Results: Among the 255 patients tested $125(49 \%)$ had at least one type of IgA autoantibody: $114(44.7 \%)$ were found to be IgA-RF positive and of these $10.5 \%$ were negative for lgM-RF and $5.2 \%$ were double negative for both IgM-RF and IgG-ACPA; thus, in these patients IgA-RF was the only detectable antibody. IgA-ACPA were detected in $79(31 \%)$ patients and apart from one exception all of them had also IgG-ACPA. Remarkably, the percentage of patients showing a SDAI50 response to TNF inhibitors was significantly lower in patients positive for IgA-RF and/or IgA-ACPA $(p<0.0001)$ compared to IgA negative patients. Thus, $58 \%$ of IgA negative (but IgM-RF and/or IgG ACPA positive) patients showed a SDAI50 response whereas only $25 \%$ of the IgA-RF and/or IgA-ACPA positive ones were responders. Interestingly, while the presence of both IgA specificities did not further change the percentage of responders, patients positive for IgAACPA but negative for IgA-RF showed the lowest response rate to anti-TNF treatment. Completely seronegative patients also showed a significantly lower SDAl50 response $(p<0.0001)$ to TNF inhibitors compared with the IgA negative (but IgM-RF and/or IgG-ACPA positive) patients. Similar results were obtained when ACR20 was used as response criteria. No differences between the various serological groups were seen with respect to treatment with MTX.

Conclusions: While the added diagnostic value of IgA antibody measurement was moderate, IgA-RF and particularly IgA-ACPA appear to be associated with poorer therapeutic responses to TNF inhibitory biological drugs and therefore may help in further stratification of RA patients and therapeutic decision making. Disclosure of Interest: None declared

DOI: 10.1136/annrheumdis-2017-eular.5210

\section{AB0218 CORRELATION OF GRAY SCALE AND POWER DOPPLER ULTRASONOGRAPHY WITH CLINICAL EVALUATION IN RHEUMATOID ARTHRITIS}

I.E. Okatan, M. Torgutalp, M.E. Yayla, A.B. Keleşoğlu Dinçer, E. Uslu Yurteri, A. Ateş, T.M. Turgay, G. Kınıkl. Department of Internal Medicine, Division of Rheumatology, Ankara University Faculty of Medicine, Ankara, Turkey

Objectives: Ultrasonography (US) is a useful method for assessing synovial vascularization and proliferation in rheumatoid arthritis (RA). The aim of the study is to compare the tender joint and swollen joint in patients with rheumatoid arthritis (RA) with gray scale (GS) and power doppler (PD) ultrasonography (US).

Methods: Thirty RA patients were included. Median disease duration was 53.7 months. Demographic and clinical data, $C$ reactive protein (CRP) level and erythrocyte sedimentation rate (ESR) were recorded for each patient. Disease activity was evaluated using the Disease Activity Score in 28-joints (DAS28) with a median score 3.8.The joint tenderness and swelling were assessed for 10 joints (wrists, second and third proximal interphalangeal and metacarpophalangeal) in each patient. These joints were evaluated by GS and PD by ultrasonography. US joint effusion, synovitis and PD signals were graded from 1 to 3 for each joints. The 10-joint GS and 10-joint PD scores were then calculated. Correlations were tested using the Spearman coefficient.

Results: GS effusion, synovitis scores $(r=0.565, p<0.001)$ and PD signals $(r=0.883, p<0.001)$ correlated highly with the corresponding swollen joints. There was a significant correlation between DAS28 and number of tender joints $(r=0.745, p<0.001)$ but no correlation was found between the tender joints and ultrasonographycal effusion, synovitis grade $(r=0.073, p>0.001)$ and the PD signal $(r=0.069, p>0.001)$. There was moderate correlation between 10 joints $G S, 10$ joints PD and DAS28, but it was not statistically significant.

Table 1. Demographic and clinical characteristics of the patients $(n=30)$

\begin{tabular}{lc}
\hline Age (years) - mean (SD) & $53.7 \pm 11.9$ \\
Female, $\mathrm{n}(\%)$ & $28(93.3)$ \\
RA duration (months) & $42(67)$ \\
Rheumatoid factor positive, $\mathrm{n}(\%)$ & $15(50)$ \\
CCP positive, $\mathrm{n}(\%)$ & $20(66.7)$ \\
Smoking, $\mathrm{n}(\%)$ & $5(16)$ \\
Prednisone, $\mathrm{n}(\%)$ & $11(36.7)$ \\
DAS28 (ESR), (IQR) & $3.8(2.9)$ \\
TJC (0-10), (IQR) & $2(5)$ \\
SJC (0-10), (IQR) & $0(0)$ \\
10 Joint GS score, (IQR) & $0(1)$ \\
10 Joint PD score, (IQR) & $0(0)$ \\
ESH mm/h (IQR) & $23.5(21)$ \\
CRP mg/L (IQR) & $4.6(6.5)$
\end{tabular}

SD: standard deviation, RA: rheumatoid arthritis, CCP: cyclic citrullinated peptide, DAS28: disease activity score in 28 joints, ESR: erythrocyte sedimentation rate, CRP. C-reactive protein, GS: gray scale, PD: power doppler, TJC: tender joint count, SJC: swollen joint count.

Conclusions: Evaluating the swollen joints with clinical examination and combining it with US is a sensitive method. As joint tenderness is a more subjective finding than the joint swelling, this may explain the lack of correlation between tender joints and ultrasonography findings. We suggest to use Gray scale US and PD as a complementary method in addition to clinical assessment of joint tenderness in patients with RA.

Disclosure of Interest: None declared

DOI: 10.1136/annrheumdis-2017-eular.4859

\section{AB0219 INTENSIVE COMBINATION THERAPY WITH MEDICATION AND ORTHOPEDIC SURGICAL INTERVENTION FOR TREATING RHEUMATOID ARTHRITIS PATIENTS WITH DETERIORATED JOINTS}

H. Ishikawa ${ }^{1}$, A. Abe ${ }^{1}$, Y. Nomura ${ }^{1}$, S. Ito ${ }^{1}$, T. Kojima ${ }^{2}$, M. Kojima ${ }^{3}$

N. Ishiguro ${ }^{2}, A$. Murasawa ${ }^{1} .{ }^{1}$ Rheumatology, Niigata Rheumatic Center,

Shibata; ${ }^{2}$ Orthopedic Surgery, Nagoya University Hospital; ${ }^{3}$ Medical Education,

Nagoya City University Graduate School of Medical Sciences, Nagoya, Japan

Background: The treatment aim of rheumatoid arthritis (RA) is achieving and maintaining remission (REM) or low disease activity (LDA) via tight medical control. However, despite remarkable advances in medication, progressive deterioration and/or deformity of the joint sometimes occurs, if adequate medication is not administered in the early stage. Surgical reconstruction is still required in the joints with functional loss caused by structural damage. Recently, patients have expressed a desire to achieve functional REM with a higher quality of life (QOL) and improved mental wellness.

Objectives: The objective of this study was to clarify the effectiveness of intensive combination therapy with medication and orthopedic surgical intervention in patients who have already achieved REM or LDA. 